\title{
Fundamental Energy-Physics and Planet Earth's Platform for Life
}

\author{
Rockley G. Boothroyd
}

Chartered Engineer, Queensland, MS537 Kingaroy, Queensland 4610, Australia

Corresponding Author Email: rockboothroyd1@gmail.com

https://doi.org/10.18280/ijdne.150201

Received: 18 August 2019

Accepted: 7 October 2019

\section{Keywords:}

nuclear fusion, nuclear stability, $2^{\text {nd }}$ law of thermodynamics, forward arrow of time, symmetry breaking

\begin{abstract}
The stability and longevity of elements in our fuel supplies is of paramount importance for the sustainability of our civilisation. The nuclear fusion fuels used in our Sun are of especial importance as almost all of our renewable energy resources derive directly from this source. In this case longevity runs into billions of years. A proposed explanation of this longevity, which applies specifically to the stability of stellar hydrogen fuel, is presented and examined. The explanation is consistent with well-established features of our general understanding of matter and quantum physics. It also has many other relevant features relating to important factors concerning life on our planet. These include: the suspected quantum-based origin of the phenomenon of forward moving time and the $2^{\text {nd }}$ Law of Thermodynamics; the, as yet, largely un-researched importance of the interaction dynamics of the up and down quarks and their attendant gluons; the suspected importance of these interactions in the fusion control dynamics of stars; and the suggestion that gravity is initiated in a similar way by these dynamic interactions which exist but are below observation level at the quantum scale. Also considered are the nature and consequences of the only Universe known to us which seems to combine both symmetrical and asymmetrical features working together harmoniously. It is also concluded that quantum-sized mechanisms are of exceptional reliability in controlling matter. Finally, it is suggested that the present world energy supply situation is reaching a more critical phase requiring a stop-gap approach to allow important superior technologies to catch up with perceived needs.
\end{abstract}

\section{INTRODUCTION}

This paper has 3 different main objectives all rolled into one paper.

a) It is a study which can be used as an example with a view to encouraging young scientific research workers and to help them get the most benefits from their own original ideas. It is a special interest of many members of our Scientific Advisory Groups at the Wessex Institute to assist Ph.D. students and young postdoctorates with their early papers.

b) This paper reviews some well-researched subjects. It seems to have been an experience similar to that of a typical research student required to enquire into new cross-disciplinary pursuits.

c) The paper reaches some new scientific conclusions relating to the forward arrow of time, the $2^{\text {nd }}$ Law of Thermodynamics, our Universe and the importance of quantum-based mechanisms which provide stability in matter. These and other matters seem to be of significance, indicating the need for further study and discussion.

\subsection{How this study started}

Initially this paper concerned itself mainly with the necessary longevity and stability of our main thermonuclear fuel $\left({ }_{1} \mathrm{H}^{1}\right)$ the lightest of hydrogen's 7 isotopes. ${ }_{1} \mathrm{H}^{1}$ is also by far the most stable of the 7 isotopes of hydrogen. Indeed, it is the most stable of all our other elements. Our Sun and other stars in our Universe will be our main source of 'so-called' "renewable energy" for billions of years. This is a time-scale which may even be necessary for us to achieve a fullyadvanced civilisation of our species. It follows that we need to ask ourselves this fundamental question which is fairly typical of a theme which is first presented to a new research student:

-"Why, and how, is hydrogen thermonuclear fuel so stable and long-lasting?"

\section{THE STABILITY OF THE PROTON}

It is a strange feature of hydrogen that it appears to be the most stable and robust of all atoms. It seems to remain stable for at least $10^{24}$ years [1] and extensive tests have failed to dispute this. It is concluded that we cannot even question the near perfect stability of the proton from which all other forms of matter are made in stellar processes [2]. Hadronisation (see section 2.1) is another amazing phenomenon, found in recent years, which is suggestive of the special nature of the proton in the hydrogen atom.

\subsection{Hadronisation}

At CERN's Large Hadron Collider (LHC) twin opposing beams of protons (in the form of hydrogen nuclei) are accelerated to within $0.000001 \%$ of the velocity of light and 
smashed together into their fundamental components which are quarks and gluons [3]. At these high velocities many different types of new exotic particles, such as pentaquarks [3] are also created from the large local energy available but these particles are all very unstable and disintegrate quickly into smaller harmless entities. These fragments are usually emitted in the form of jets [3] as illustrated in plates 1-2 in the book [1]. It is found that if a quark is knocked away from its position in the nucleus, a gluon will pull it back into position. If, as is often the case, the impact is severe enough to detach the quark completely from its nucleus, the gluon will appear to stretch. If the gluon breaks under the strain, it will produce another gluon and then another gluon is generated, and so on [3] as is needed under these conditions. In this way these high energy impacts can generate new quark and antiquark pairs which can then recombine quickly to form new hadrons including new hydrogen nuclei.

This is a most remarkable and effective self-repair mechanism and it goes a long way in explaining the robust nature of ${ }_{1} \mathrm{H}^{1}$. Hydrogen, anywhere in space, can be exposed to some very energetic natural cosmic rays causing damage to the proton at any time. It follows that there is a fundamental need for the hadronisation phenomenon to exist.

We have no comparable repair system in any of our normalsized engineering systems. We know [4] that when a gluon is very close to a quark the force between a gluon and quark is quite small but it increases strongly to a high constant value when any separation occurs. It behaves like the effect of a spring-loaded detent ball in the notch of an operating shaft in an automobile gear box but the similarity ends there. No earthbound engineer could invent a gear box which can repair itself while still working. The hidden technology within the hydrogen nucleus seems superior to anything known to us.

An eminent physicist (the late Richard Feynman) described the components within the proton as partons which includes quarks and gluons. On the basis of the evidence from section 2.1 alone, a more descriptive term for the collective interaction of partons might be to call them quantum-sized mechanisms (QSMs). To avoid any confusion, it might also be better to try to avoid the term quantum mechanics and restrict ourselves to the term quantum physics.

Bosons, such as gluons, are completely different from fermions, such as electrons. Bosons have much freedom of movement and are not subject to the Pauli Exclusion Principle as is the case with fermions but both acts together to give us the stable matter system we know. The mechanisms behind the Pauli Exclusion Principle (PEP) are also mainly one of the amazing unexplained mysteries of modern physics. PEP can also be described as a particularly complicated form of QSM and is discussed below in Appendix IIc.

Finally, it seems worthwhile to comment that Nature seems to be parsimonious regarding the conservation of hydrogen atoms. Conversely Nature seems to be recklessly extravagant in so many other areas. The vast size of our mostly inhospitable Universe is one such example. Another such example of extravagance is the wasteful mechanism of life's evolutionary development itself as expressed by Charles Darwin.

\section{THE INNER STRUCTURE OF THE PROTON AND NEUTRON}

The hydrogen atom (in the form of a quark-gluon plasma [5] appears to have been the fundamental form of matter at the beginning of time following the supposed "Big Bang" event. Other elements are made from it by a range of nuclear fusion events [2]. In Mendeleev's Periodic Table, hydrogen seems to be an oddity when compared with associated elements such as Lithium, Sodium and Potassium etc. This feature also raises our suspicions regarding hydrogen's special nature.

The hydrogen atom nucleus is composed of 3 valence quarks which interact with each other and also their attendant gluons. For an explanation of valence see Appendix I. The gluons are a form of particle called gauge-bosons. Photons are another form of boson but they differ greatly from gluons. The gluons bind the nucleus together with the "strong force" to give a very stable atomic nucleus which resists the powerful electrostatic force within the quarks themselves. This electrostatic force tries to explode the nucleus apart. This may, on a first impression, appear to be a very strange way in which to create matter but it is the only system known to us (but see Appendix IIb where suspected 'fine tuning' may offer an explanation). To the best of the present writer's knowledge no scientific team has ever attempted (from a purely theoretical standpoint) to design an alternative form of basic matter which might be very different, perhaps even superior, to the matter we know. The reason for this is obvious enough. We do not yet have the basic knowledge to embark on such an ambitious enterprise.

The available scientific literature also seems to have given no serious consideration to the fact that the number of 'valence' quarks in a hydrogen nucleus is 3 . Yet in this paper it is suggested that this seems to be a number of much significance.

It is also of great interest that a neutron which is outside the nucleus is unstable, decaying into a proton with a half-life of about 600 seconds. Yet when a free neutron penetrates and becomes part of the nucleus it gives stability both to itself and to the new isotope's nucleus. We can conclude that the neutron has an inherent stabilising effect but only when it is within the nucleus. Also, the other components within the nucleus seem to contribute to this same stabilising effect.

It seems significant that protons have 2 'up' quarks and 1 'down' quark. On the other hand, neutrons, also comprise 3 quarks, but in this case, there is only 1 'up' quark but there are 2 'down' quarks. Quarks carry a different form of charge which is called 'colour' charge. These charges on the 'up' quark have a value which is $2 / 3$ of that carried by the electron but the charge is positive whereas the 'down' quark has $1 / 3$ of the value of that carried by an electron and it is also a negative charge. Simple arithmetic shows that the charge carried by a proton is one electron unit but it is positive, whereas the neutron has no net charge. It is important to note that colour charge on a quark always behaves as a vector quantity mathematically whereas the unit charge on an electron behaves as a simple scalar quantity unless it is carried by a moving particle when it automatically becomes a vector. It would be presumptuous of us to believe that there is a close relationship between charge in a quark and charge carried by an electron. The whole subject of electrostatic charge is not well understood at the fundamental level but clearly there are rigid rules relating to charge in QSMs.

For elements with a higher number of protons in the atom, a neutron/proton ratio slightly higher than 1 tends to give more stable isotopes. By a mechanism which is not understood an excess of neutrons can promote a slightly stronger nucleus where the stresses within larger nuclei become too oppressive. This is a reasonable expectation as the gluons in these larger 
atoms are more highly stressed in resisting the larger disruptive force of the higher electrostatic force in these larger nuclei.

\section{SELF-TESTING AND REPAIR (STAR) SYSTEMS IN OUR OWN TECHNOLOGIES}

Quarks and gluons are particularly troublesome for experimenters because they cannot be isolated for examination by themselves because of the need for 'confinement'. If we take a quark out of its nucleus it just disintegrates before we can examine it. This is suggestive that quarks and gluons have hidden inner mechanisms.

It seems reasonable to suspect that the combination of 2 'up' quarks and one 'down' quark is a very special combination regarding stability.

The suspicion that 3 quarks can achieve this exceptional stability also suggests a similarity with STAR (self-testing and repair) computers which we have used on deep space missions where absolute computer reliability is needed. In deep space, where radiation is strong, damage to electronic equipment is a prominent hazard. Quite often when we develop a clever technology like STAR computers, we then find that Nature has already been using similar methods for aeons.

\subsection{STAR computers, their principle of operation and suspected similarity with quark-gluon protonic behaviour}

Usually our STAR control systems used in deep space missions comprise 3 computers working in parallel and they work simultaneously on the same calculations. After a sufficiently short repeated-time interval $\mathrm{T}$ of operation, the 3 computers compare their results. It is then easy to locate the source of an electronic malfunction by an electronic 'voting' system. By comparing the 2 computers which agree with the computer which differs it is possible to identify which computer is at fault. An electronic switch can then be triggered to insert a spare integrated circuit to replace the one which is suspected of failure.

For the sake of generality let us assume that the probability of a malfunction of each of the computerised stages of calculation is $\mathrm{P}_{1}, \mathrm{P}_{2}$ and $\mathrm{P}_{3}$, although invariably $\mathrm{P}_{1}=\mathrm{P}_{2}=\mathrm{P}_{3}$ because the computers must be identical. If the time interval $\mathrm{T}$ between comparisons is sufficiently short and $\mathrm{P}_{1}, \mathrm{P}_{2}$ and $\mathrm{P}_{3}$ are then very small probabilities much less than unity, then analysis can then proceed usefully.

The probability of any two of the computers giving the same error is $\mathrm{P}_{1} \times \mathrm{P}_{2} ; \mathrm{P}_{2} \times \mathrm{P}_{3}$ and $\mathrm{P}_{1} \times \mathrm{P}_{3}$ respectively.

Inserting some reasonable values such as: $\mathrm{P}_{1}=\mathrm{P}_{2}=\mathrm{P}_{3}=10^{-8}$, then normally if there is no fault $\mathrm{P}_{1} \times \mathrm{P}_{2}=10^{-16}$ etc. for all 3 comparisons. Thus, there is only a 1 in $10^{8}$ chance that the faulty component will fail to be identified correctly. An automatically programmed repeat of the test can reduce the chance of failing to detect the fault location as 1 in $10^{16}$. For very important systems a further repeat test can reduce the uncertainty to 1 in $10^{24}$.

There are a number of alternative mechanisms of the above type which can be used to make computers totally reliable and able to repair themselves. Using the above example if the two 'up' quarks in the proton can introduce into the 'down' quark (also incorporating its attendant gluon) a temporary copy of one of the up quarks then a totally reliable checking system is possible to ensure that the correct state of each quark exists in the hydrogen atom. As detailed in Appendix I there is ample evidence of a very high level of unexplained activity within the proton's quark structure which is suggestive of such activity.

If this is the case, then the important point to note is that a constantly repeating time interval generator for $\mathrm{T}$ would exist somewhere within the quark trio and $\mathrm{T}$ must be small enough to achieve the necessary small values of $\mathrm{P}_{1}, \mathrm{P}_{2}$ and $\mathrm{P}_{3}$. Also, as a quantumised feature, the value of $\mathrm{T}$ would be identical for all protonic matter. Thus, every element in Mendeleev's periodic table would have the same mechanism and hence the same value of $T$ would be seen in every other element. In short, the value of $\mathrm{T}$ would be a universal one in all matter. This is consistent with our expectations.

The supposition that the quark trio is a generator of a fixed time interval suggests that the origin of time as we know it would most likely lie within the 'up' quark duo in the quark trio of the proton. If this is the case then forward moving time, as we know it, has its origins in all forms of matter as a quantum effect. This is a reasonable suspicion because all sizeable matter has a proton content and all matter appears to experience forward-moving time in the same way. Also introducing a forward moving time generator within the proton implies the automatic introduction of a system whereby sequential cause and effect can take place. Only when this is so can chemical reactions then take place. If chemical reactions can take place then the ultimate emergence of lifeforms is possible. This suggests a mechanism whereby the Second Law of Thermodynamics comes into our World of existence. All this would seem to explain away much of the puzzling problems [6] we have experienced concerning the nature of our strange Universe. In this earlier paper [6] it was surmised by the author, and also by other researchers, that forward-moving time might result from quantum decoherence. However, the present author has made no progress at all with this concept. It seems that quantum decoherence is insufficiently precise to account for the constant stability of forward-moving time. Despite this setback it still seemed reasonable to suspect that another aspect of quantum physics could explain the mechanism behind the forward arrow of time.

This suggestion concerning the quantum-based origin of forward-moving time is, perhaps, the most important, yet tentative, conclusion of the present paper. It is therefore a suggestion which requires much more examination, particularly at the experimental level.

STAR systems are now quite commonplace in our world where we require absolute reliability such as in the safety of airliners. Alternative configurations of reliable systems are classified in the literature under the following subject headings such as: redundancy engineering, reliability engineering and fault tolerance.

\section{EXPERIMENTAL INVESTIGATING BEHAVIOUR \\ TECHNIQUE FOR
SITU, QUARK-GLUON}

Because the working behaviour of quarks and gluons can only be examined when they are 'confined' within the nucleus, more precise measurement techniques are required than is possible by merely colliding particles together by brute force.

The most relevant is the use of electrons of high but known energy directed at the atom's nucleus in a method called deeply virtual Compton scattering [4]. Data of particular 
events are measured and kept separate by using conventional electronic coincidence techniques. Several other papers have been written in this subject area which is a useful one although the method seems to need much time and effort to get results.

There are variations on this experimental approach, notably deep inelastic scattering (DIS) which has been reviewed recently [7] showing that many of our theories do not yet agree with more reliable experimental data.

\section{OTHER RELEVANT MATTERS}

Several other important matters, for which there is limited information, stem from the present study and just three of them are listed briefly in Appendix II. The following $4^{\text {th }}$ matter, which is of major personal interest to most of us, is examined in sections 7 and 8 .

\section{DISCUSSION RELATING TO ASYMMETRY IN OUR UNIVERSE AND IDEAL SYMMETRICAL UNIVERSES [8]}

It is reasonable to ask ourselves why we have not already discovered the apparent importance of a quantum-based mechanism for generating the forward arrow of time and the reasons for the incorporation of the related Second Law of Thermodynamics into the physical behaviour of our Universe. A possible explanation may be along the following lines: -

\subsection{The standard model of particle physics and QCD}

The Standard Model of Particle Physics [3] can be considered to be the most outstandingly successful example ever achieved in the development of a valuable but empirical model. The Standard Model uses 26 different arbitrary parameters and all of them are found to be relevant. However, in many cases we are not sure how and why these parameters work [3]. Also, sometimes the parameters have to be determined numerically by experience alone in order to agree with the data we observe.

Perhaps, as a likely consequence of this, the Standard Model has been found to be so accurate in its predictions that many physicists would prefer it to be less accurate so that it could indicate promising new lines of research to improve our realistic understanding of matter [3].

Quantum Chromodynamics (QCD) [9] is an integral part of the Standard Model. QCD was developed from an earlier and very successful but far less complicated theory called Quantum Electrodynamics (QED).

Both QCD and QED are based on Symmetry [3, 8]. If our Universe was a symmetrical one [8], then time as we know it, would also be able to run backwards as well as forwards. We would not experience thermodynamic irreversibility and entropy would not be able to increase with passage of time Such a universe would be an oversimplification of the real Universe in which we live. Also matter, as we know it as a cause of irreversibility, could not exist in a symmetrical universe. Thus, we have no option but to realise that QCD has its limitations when studying our own Universe. Nevertheless, as explained in section 8 , the idealised symmetrical universe is of exceptional interest to us for theological reasons. Thus, it is expected that QCD [9] would seem to be a useful tool for examining the idealised symmetrical universe.

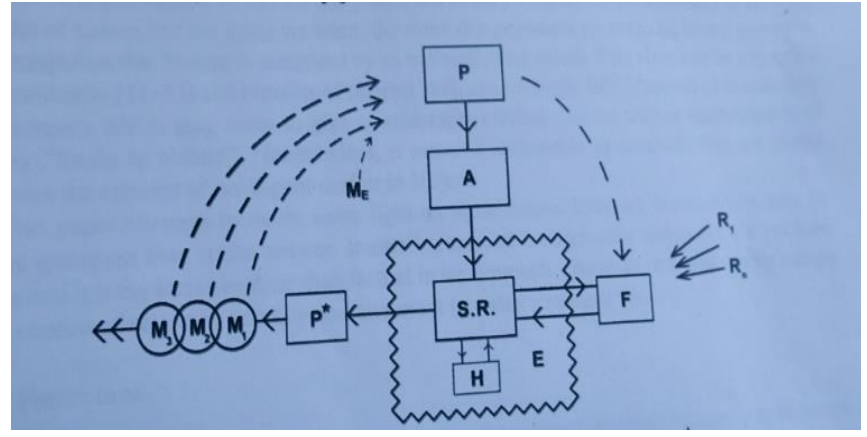

Figure 1. Time future, present and past [6]

In Figure 1 [6], $\mathrm{F}$ is the future which can only become apparent to us in the present instance $\mathrm{E}$, the future can also depend on random effects $R_{1}-R_{n}$ due to the uncertain statistical nature of quantum physics. A was originally thought to be from the quantum decoherence effect but is now explained in section 4 of the present paper. $\mathrm{P}$ is true time which we would expect in a true fully symmetric external universe where there is only elastic behaviour, no entropy increases and full reversibility. This is considered to be the same state as $\mathrm{P}^{*}, \mathrm{M}_{1}$, $\mathrm{M}_{2}, \mathrm{M}_{3}$ etc. in the past after it is leaving the present instant.

Paul Davies [10] has very aptly described our planet as a "Goldilocks" planet but it is only a mere speck in our expanding Universe now extending to about 50 billion light years in size. Moreover, it is apparently the only universe known to us. Thus, our Universe is the only one which we can see and examine, despite widespread belief of many scientists in the likely existence of other universes besides our own. Perhaps we should describe our Goldilocks planet as part of our "Goldfish Bowl" Universe? Even if goldfish cannot reason about their existence, they can at least see a world outside their own bowl. We humans seem even more incapacitated: unlike goldfish we may be able to question our existence but we cannot see anything outside our Universe with which to make comparisons. Or can we? Section 8 may throw some light on this difficulty.

\section{THEOLOGICAL CONCLUSIONS}

Section 4.1 suggests that the phenomenon of forward moving time has a physical basis at the quantum level which has its origin from within the protons of matter. If this can be shown to be confirmed by future experiments, it is merely a physical effect deriving from the behaviour of matter. Its fundamental purpose was presumed in an earlier paper [6] to create a world in which life forms can develop. The fundamental effect of forward moving time is also conditional upon the necessary asymmetric nature of the Universe in which we live.

Yet it seems that we can suggest, from the following scientific reasoning, that an alternative universe which is symmetrical might also be able to support a different kind of life-form. The model of time development was illustrated in Figure 1 in the paper [6] and is reproduced here as Figure 1 for convenience. In this diagram the wavy section $\mathrm{E}$ is the universe in which we live. To be more realistic, the wavy rectangle $\mathrm{E}$ should be drawn microscopically thin as 2 coincident vertical wavy lines. This is because it represents the tiny increment of time in which we exist from moment to moment. Figure 1 was also deliberately drawn originally in a vectorially-incorrect manner with the increment of time being 
illustrated as stationary so that it could be examined in this earlier paper [6]. From moment to moment, E changes and in reality, it can be considered to move to the right as time moves forward leaving the past $\left(\mathrm{P}^{*}\right.$ and $\mathrm{M}_{1}$ etc.) behind and stationary in the relative sense. This is the effect which appears to give us the false impression that time appears to flow. Each increment of the past $\left(\mathrm{M}_{1}, \mathrm{M}_{2}\right.$ etc.,) is lost to us although we can keep records $(\mathrm{H})$ of what becomes the past. These records are always with us but they are only available to us in the present. To us the past is just a memory. Our memories have no mass and are invisible to us except as memories, be they in our minds or in more permanent form of records. Our past has apparently left us and seems to be outside our own immediate Universe. It seems reasonable to consider the past as a series of multiverse-type past events outside our own instant $E$ of existence.

Our experience in physics such as a symmetrical universe becoming an asymmetrical one is known as symmetry breaking. Any form of symmetry breaking seems to be unusual [8] but it can be surmised that symmetry breaking may be for a directed purpose to achieve an unusual objective. On the other hand, a symmetrical universe would be expected to contain no matter at all but only considerable energy [11] which unfortunately is not available for our immediate use. Even in our own asymmetrical universe it is the Higgs boson and its field which is supposed to account for all mass. The valence quarks and gluons in Table 1 in Appendix I would have zero mass if there was no Higgs boson, in other words all the non-energy mass they exhibit is attributed to the Higgs field permeating all space from the Higgs boson. In a symmetrical universe all phenomena would be elastic and fully reversible. Apart from being sterile [6] a symmetrical universe could only contain life-forms which do not contain matter, i.e. any living creatures, if they exist, could only be spiritual in nature. Also, because a symmetrical universe is fully reversible, such spiritual life-forms, if they exist, could be expected to be able to live for all eternity and move backwards and forwards in time at will.

Physical matter which changes its form and animal life, such as ourselves, cannot move backwards in time because of Prigogine's entropic barrier [12,13]. This same limitation would appear to be true for any physical creature trying to visit us from the future by coming into our own moment of time. The lack of tourists from the future and our own inability to visit our past, which would create a serious paradox, seem to confirm all this. Anyway, the fundamentals of quantum physics suggest that there is no such thing as a deterministic future for us until it becomes part of the present instant in which we live. This is because of decoherent collapse of all but one set of wave-functions deriving from Schrodinger's equation. All the non-final wave-functions of this equation decohere to equate to what exists in the present instant. This, in fact, is how we try to plan our lives, namely by planning a future instant so that decoherence will take place in line with our future needs and not according to the arbitrary random probabilistic decisions of quantum physics.

Yet when we consider the disappearing past as a separate but symmetrical entity (i.e. separate universe) outside our own universe then the idea of a continuously developing separate universe seems to be reasonable. Such a symmetrical universe might contain spiritual beings which could exist for all eternity. Also, because such massless beings are not limited by Prigogine's entropic barriers, they would be able to move from one of these separate universe increments to another. It seems that this instance, when Prigogine's entropic barrier cannot operate, makes the development of such a separate multiuniverse system possible. This raises the question of a possible form of afterlife.

It must be emphasised that this may not be the only possibility of creating a form of afterlife but it does seem to suggest that the doctrines of our monotheistic religions concerning an afterlife are reasonable ones and not just wishful thinking.

There is an important consequence of the above suggestion. If our own existence on earth is limited to using our senses in an asymmetric universe where matter exists, then outside this personal universe, the rest of our universe may be symmetrical and potentially fully time-reversible. This raises the conclusion that our Universe seems likely be a composite one with both symmetrical and asymmetrical features which coexist together in harmony. Where there is matter our Universe is asymmetrical with a forward arrow of time. In the much bigger volume of space which is almost empty except for occasional mere specks of matter, (which limits the decoherence effect) this part of the universe would appear to be able to behave in a symmetrical way.

Strong evidence for such a composite universe is that we experience both elastic and inelastic forms of scattering by fundamental particles. Elastic, i.e. thermodynamically reversible scattering is part of a symmetrical universe. Inelastic scattering i.e. thermodynamically irreversible scattering is part of an asymmetrical universe. Moreover, the fact that these different forms of scattering can occur in close proximity suggests that our Universe is not only a composite one but it is one where symmetry and asymmetry can coexist together on a very small and intimate scale.

The moment of time, (E) in Figure 1 which is just a vertical line if considered realistically, in which we perceive our environment is effectively life in a very thin personal membrane but it seems to be one, which although tortuous, is connected with other parts of our Universe where matter exists Our personal asymmetric Universe is a very small one but it enlarges considerably when we share it with other creatures. Isolated spaces containing matter and hence asymmetrical forward-moving time exist but they are based on the same mechanism of time generation and they can communicate with other similar isolated asymmetrical spaces. Appendix III contains a sequel to an earlier study [13] which supports the above concepts.

If our Universe is a composite one as suggested, would this help with finding more convincing explanations of the nature and existence of dark matter and dark energy?

\section{CONCLUSIONS FOR YOUNG PH.D STUDENTS}

There is plenty of good advice on the Web for research students concerning the many problems they have to face. Much of the additional advice here is self-evident from reading this paper. However, three factors require further comment.

\subsection{The problem of 'mind-set' and creativity}

Often the first year or so of your work is largely devoted to searching the relevant literature. It seems advisable to avoid the problem of 'mind set' which can limit your creativity. It is suggested that literature searching may best be divided into a preliminary stage where notes are made of the student's own 
ideas which are written down for possible future development. Usually these ideas may seem less promising as your research progresses but sometimes this may not be the case. A more detailed literature examination can then follow later. An uncluttered mind seems vital in modern research because our world is now very complicated. Interesting ideas by other researchers often result in 'dead ends'. Sometimes the dead ends can be avoided by taking a different path and these alternatives may be written down in your earlier notes.

\subsection{The need for cross-disciplinary attitudes}

To some readers this description of the research subject in this paper may seem to have been a lucky one in having so many interesting features. To a very large extent this may be true but is a result of explorations across the boundaries into other scientific disciplines.

It is now more than 50 years since the present writer graduated as $\mathrm{Ph} . \mathrm{D}$. As a young researcher his task was to help to develop a new type of more efficient gas cooled nuclear reactor. Ample research funds were available. These were heady days when British and American industries and their government departments were keen to develop commercial nuclear power which was also the way to manufacture Plutonium-239. This was easier than enriching natural Uranium to $90 \%$ weapons grade Uranium-235. Supposedly this enabled these two nations to advance their international status by mass-producing nuclear weapons using Plutonium!

Unfortunately, 18 months into the project it was discovered by the present author that a fundamental and uncorrectable flaw in the design concept would make the new reactor very dangerous. Fortunately, boundaries into other scientific disciplines had already been explored and the first 2 articles by the present author were published under the auspices of the British Institution of Chemical Engineers, a learned professional body for which the present writer has never had formal qualifications. It seems that research students can be either very lucky or very unlucky but if you end up in the latter category there is still merit in publishing adverse findings. It may help another researcher to avoid your misfortune. Experience and knowledge are always valuable.

Cross-disciplinary experience is worth the effort and will usually improve the graduate's future employability. A widely experienced problem-solver can always get a job in today's complicated world.

\subsection{A perspective on future energy research}

Our ability to supply a large and increasing world population desirous of energy for a better life depends critically on developing new stop-gap technologies which may only have value for a limited time. Wind and solar farms have made excellent progress and are very important but they are only a limited answer as they compete with valuable agricultural land. Even the most ardent climate-change deniers are now being forced to accept that most of our fossil fuels must be left in the ground at least as an interim measure. Climate change is a growing threat to our civilization. Nuclear fusion is our long-term hope but our two main technologies (electromagnetic plasma and inertia confinement) are extremely difficult to commercialise. We cannot use the necessary high fusion pressures needed within stars and our remaining chance (muon-catalysed fusion) to overcome this problem seems likely to remain just a laboratory curiosity and even more difficult to commercialise. Transport problems in nations of large area like U.S.A., Russia Canada and Australia seem to be particularly troublesome but we seem to have ways to alleviate this. All this will leave a gap in the necessary supply of energy.

It seems to the present writer that an updated nuclear fission reactor industry is needed. The reasons for going back to nuclear fission as a stop-gap resource are argued along the following lines. The molten salt nuclear fission reactor in fast neutron form seems to meet this requirement [14]. It is a compact and very safe system. While it works breeding new fuel from ample Thorium and Uranium reserves it burns up long lived radioactive waste which then needs only 300 years to store the waste when it can be returned to the environment after extracting valuable transmuted trace elements which are normally only created in stellar supernovae. This form of energy can give us a resource which can last nearly 1000 years. These reactors operate at atmospheric pressure and work at sufficiently high temperature to be efficient and also separate steam generation completely from nuclear fission with an intermediate heat exchanger. Water was always dangerous when used inside nuclear reactors.

\section{GENERAL CONCLUSIONS}

\subsection{The origin of forward moving time}

Forward moving time is fundamental to the existence of the Second Law of Thermodynamics. It has long been suspected that it has a scientific basis related to physics at the quantumsized level. It is concluded that it is not related to quantum decoherence but appears to be related to interaction dynamics between 'up' quarks and gluons. In the absence of any other plausible explanation for the forward arrow of time, further study is recommended when appropriate experimental techniques can be developed.

\subsection{The importance of quantum physics in the control and organisation of natural physical phenomena}

The longevity (and perhaps even its generation) of hydrogen through hadronisation is an example of a well-organized quantum-sized mechanism (QSM). Other QSMs such as the Pauli Exclusion Principle exist. Even Heisenberg's uncertainty principle seems likely to be a QSM. QSMs are characterised by being control systems with a high level of reliability [15] thus giving us matter of high stability. This is the other and neglected arm of quantum physics which is often merely described as the science of uncertainty [16].

\subsection{The nature of our composite universe}

The only Universe we know appears to have a composite nature of both symmetrical and asymmetrical features which work together in a harmonious and closely integrated way. There are some grounds to support the suspicion that it exists not only to support life of physical biological creatures such as ourselves but also it can exist as a support system of massless forms of life which we would describe as being only spiritual in nature and thus inaccessible to us. 
10.4 Past time as a likely origin of a series of multiverses of theological significance

Past time is the most mysterious aspect of the forward moving time phenomenon. Yet it is the most tangible aspect we have of multiverse theory which is a subject taken seriously by many of today's scientists. This form of multiverse is a completely symmetrical entity where time can move backwards as well as forwards and where there is no matter but only energy and no entropy changes.

\subsection{Policy matters relating to present-day energy supply problems}

The problem of providing sufficient energy for our world without causing environmental damage is becoming more critical. In particular we seem to need stop-gap alternatives such as updated nuclear fission technology to enable us to find our way to the fusion power era which is still far away in the distant future.

\section{REFERENCES}

[1] Wilczec, F. (2010). The Lightness of Being: Big Questions, Real Answers. Penguin Books Ltd, Penguin: London, 292 pages.

[2] Dinwiddie, R., Rees, M.J. (2012). Universe: The Definitive Guide. 2nd Edn., Dorling Kindersley: London, ISBN: 0756613647.

[3] Hesketh, G. (2016). The Particle Zoo: The Search for the Fundamental Nature of Reality. Quercus: London, 1-322.

[4] Burkurt, V.D., Elouadrhiri, L., Girod, F.X. (2018). The pressure distribution inside the proton. Nature, 557: 396399. https://doi.org/10.1038/s41586-018-0060-z

[5] Rafelski, L., Birrell, J. (2014). Traveling through the Universe: Back in time to the quark-gluon plasma era. Journal of Physics Conference Series, 509(1). https://doi.org/10.1088/1742-6506/509/012014

[6] Boothroyd, R.G. (2017). The Heraclitian nature of time in the platform for life and its relationship with Parmenidian reality. International Journal of Design \& Nature and Ecodynamics, 12(2): 143-155. https://doi.org/10.2495/DNE-V12-N2-143-155

[7] Geesaman, D.F., Reimer, P.E. (2018). The sea of quarks and antiquarks in the nucleon: A review. Nuclear Experiment. https://doi.org/10.1088/1361-6633/ab05a7

[8] Lederman, L.M., Hill, C.T. (2004). Symmetry and the Beautiful Universe. Prometheus: New York, 1-363.

[9] Wilczec, F. (2000). QCD made simple. Physics Today, 53(8): 22-28. https://doi.org/10.1063/1.1310117

[10] Davies, P. (2008). The Goldilocks Enigma: Why is the Universe Just Right for Life? Houghton Mifflin; New York, 1-315.

[11] Krauss, L.M. (2012). A Universe from Nothing: Why There is Something Rather Than Nothing, Simon and Schuster: London, 1-202.

[12] Prigogine, I., Stengers, I. (1984). Order out of Chaos: Man's Dialogue with Nature. Bantam: Toronto, 257-296.

[13] Boothroyd, R.G. (2015). Non-relativistic time, existence and adaptation. International Journal of Design \& Nature and $\quad$ Ecodynamics, 10(3): 199-212.
https://doi.org/10.2495/DNE-V10-N3-199-212

[14] Boothroyd, R.G. (2017). Nuclear power can be the answer. International Journal of Energy Production \& Management, 2(3): https://doi.org/10.2495/EQ-V2-N3-225-238

[15] Shi, H., Milloti, E., Bartalucci, S., Bazzi, M., Bertolucci, S., Bragadireanu, A.M., Cargnelli, M., Clozza, A., De Paolis, L., Di Matteo, S., Egger, J.P., Elnaggar, H., Guaraldo, C., Iliescu, M., Laubenstein, M., Marton, J., Miliucci, M., Pichler, A., Pietreanu, D., Piscicchia, K., Scordo, A., Sirghi, D.L., Sirghi, F., Sperandio, L., Vazquez Doce, O., Zmeskal, Z., Curceanu, C., Widmann, E. (2018). Experimental search for the violation of Pauli exclusion principle. The European Physical Journal C, 78 319. https://doi.org/10.1140/epjc/s10052-018-5802-4

[16] Anon. (2017). The Quantum World: The Disturbing Theory at the Heart of Reality. New Scientist (John Murray Learning): London, 1-223.

[17] Reimer, P.E. (2016). Sea quarks in the proton. 21st International Conference on Few-Body Problems in Physics, 113(05012): 1-4. https://doi.org/10.1051/epjconf/201611305012

[18] Siegel, E. (2017). Why does the proton spin? Physics Holds A Surprising Answer. https://www.forbes.com/sites/startswithabang/2017/04/0 9/why-does-the-proton-spin, accessed on Jan. 17, 2020.

[19] Tu, Z., Kharzeev, D.E., Ullrich, T. (2019). The EPR paradox and quantum entanglement at sub-atomic scales. High Energy Physics - Phenomenology (hep-ph). https://doi.org/10.1103/PhysRevLett.124.062001

[20] Friederich, S. (2017). Fine-tuning. Stanford Encyclopaedia of Philosophy.

[21] Dawkins, R. (2006). The God Delusion. Penguin: London, 1-471.

[22] Gschwendtner, E., Adli, E., Amorim, L., Apsimoncj, R., Assmanne, R., Bachmann, A.M., Batsch, F., Baucheb, J., Olsen Berglyd, V.K. Bernardinib, M., Binghamo, R., Biskupbd, B., Bohlb, T., Braccob, C., Burrowsiw, P.N., Burtc, G., Buttenschönm, B., Butterworthb, A., Zhangct, H. (2016). AWAKE, the advanced proton driven plasma wakefield acceleration equipment at CERN. Nuclear Instruments and Methods in Physics Research: Section A, Accelerators, Spectrometers, Detectors and Associated Equipment, $\quad$ 829: 76-82. https://doi.org/10.1016/j.nima.2016.02.026

\section{ACRONYMS}

CERN Conseil Europeen pour la Recherche Nucleaire, Near Geneva

DIS Deep Inelastic Scattering

LHC Large Hadron Collider located at CERN

PEP Pauli's Exclusion Principle

QCD Quantum Chromodynamics

QED Quantum Electrodynamics

QSM Quantum-sized mechanism. (a term which is used to describe organised partons acting together in an ordered procedure.)

STAR Self testing and Repair (of computer systems) 


\section{APPENDIX}

\section{APPENDIX I: EVIDENCE FOR THE HIGH LEVEL OF ACTIVITY OF QUARKS AND GLUONS WITHIN THE PROTON}

As mentioned in section 2.1 when a proton is seriously damaged by a high energy particle, the quark/gluon mechanism generates quark/antiquark pairs which in a short time later will produce new atoms of hydrogen. Thus, there are more than the original 3 (valence) quarks within the nucleus of the hydrogen atom which has just been damaged. These extra quarks are known as 'sea' quarks. The normal way to try to understand the details of this QSM is to use the DIS method of examination which unfortunately cannot discriminate between quarks and antiquarks [17]. Is the damaged quark simply replaced with a newly-produced quark or is the mechanism of repair more complicated? All we seem to know is that there is a lot of activity within the nucleus. The 3 valence quarks communicate with each other all the time and each one has one of 3 states called colours which alternate. The term 'colour' is just a term of classification and has nothing at all to do with colour as we see it. Each valence quark always has just one of the 3 colours but the 'summed up' nucleus is always colour neutral. Colour conservation is always present just as 'spin' and energy/mass are also conserved.

Also, the quarks communicate continually with the gluons in the nucleus and gluons can have any one of 8 'flavours'. The gluons also communicate with each other and this 'flavour' classification (which has nothing to do with taste) is also a conserved quantity.

The conclusion is that we do not yet understand the details of hadronisation which may be much more complicated than the basic system described in section 4 .

Rather than trying to unravel the mathematics of various theories of hadronisation it may be more instructive to consult the paper [18]. This is a collection of pictures from research in laboratories such as CERN and Brookhaven illustrating the supposed interaction dynamics within the proton's nucleus.

Table 1. Comparison of the interactive activity of the three valence quarks (i.e 'up' and 'down') with other quarks which are not major contributors to mass in the Universe [Source: Wikipedia 2019)]

\begin{tabular}{|c|c|c|c|}
\hline Type of quark & $\begin{array}{c}\text { Total mass } \\
\text { of 'naked' } \\
\text { quark }\end{array}$ & $\begin{array}{c}\text { Total mass of } \\
\text { 'dressed' } \\
\text { quark }\end{array}$ & Relevance? \\
\hline Up quark & $1.9-2.8$ & 336 & yes \\
\hline Down quark & $4.3-5.2$ & 340 & yes \\
\hline Strange quark & $92-104$ & 486 & no \\
\hline Charm quark & 1300 & 1550 & no \\
\hline Bottom quark & 4200 & 4730 & no \\
\hline Top quark & 50000 & 77000 & no \\
\hline
\end{tabular}

Finally, it is of value to examine the data in Table 1. The difference in mass values for the up and down quarks in a proton is very large indicating that most of the effective mass is associated with much energy activity. The numbers for the charm, bottom and top quarks are almost identical indicating that there is no similar activity. The author should point out that details of quark-gluon interaction dynamics is beyond our present understanding. Also, some of the assumptions used in acquiring the data in Table 1 are open to question. In particular the present author supports the belief that communication between quarks and gluons is based on quantum entanglement [19].

N.B. All masses are stated as their energy equivalence $\left(\mathrm{Mev} / \mathrm{c}^{2}\right)$ according to Einstein's equation $\mathrm{E}=\mathrm{mc}^{2}$ where $\mathrm{m}$ is corrected for relativity effects. Strictly speaking the terms $\mathrm{m}$ and $\mathrm{E}$ are for incremental changes as are used here. The above numbers are approximate and may be updated in later editions of the source cited. A naked quark mass is the 'at rest' mass which is attributed to the Higgs field. A dressed quark also includes the energy related to activity. Only the up and down quarks contribute significantly to matter as we know it which is composed mainly of protons and neutrons. In fusion reactors a large part of the energy released is from the dressed quark mass.

\section{APPENDIX II: OTHER RELEVANT FACTORS IN THIS STUDY}

AII(a): Hadronisation inside the centre of stars

It is not commonly realised that the heat from thermonuclear fusion in the core of a star can take more than 10,000 years to reach the stars surface and be radiated into space. Because most stars function reliably this implies that the fusion control systems within the star must exist locally where fusion takes place. Pressure from gravity is certainly important because it is known that small stars burn up their fuel much more slowly than big stars. It also seems likely that the hydrogen quarkgluon plasma in a star must be subject to intense radiation damage which is self-repairing so that one can expect an additional control system at work with enough negative feedback to help control fusion. The quark/gluon/ hydrogen plasma is in the region of 'asymptopic freedom' regime [9] where all nucleon components can move freely without 'confinement'. Writing and solving heat transfer equations (with gamma ray transfer considerations etc.) and solving them numerically to see that they agree with observations seems feasible enough except that we do not yet have knowledge of accurate data to use in our equations.

\section{AII(b) Fine tuning}

Fine tuning is the observation that many of the parameters which describe our Universe must be within close limits otherwise we and our Universe could not exist. The scientific community's opinion is divided on this matter particularly when the fine tuning argument is extended to the anthropomorphic argument that only a grand designer such as God could be responsible for Creation as we see it. This whole subject is covered in an extensive reference [20] which is revised as our knowledge increases.

It seems to the present author that the more we learn, the stronger is the evidence for fine tuning. Perhaps the argument can best be settled ultimately along the following lines: -

Imagine a scenario where our species becomes extinct and another intelligent species on an archaeology trip finds and examines some of our old machines. They would discover that some of our design details had a 'multiple purpose'. For example, a steel sheet pressing such as from an old automobile might protect another important and fragile component such as a hose for hydraulics; also give adequate strength for the whole structure; minimise vibrations which could cause fracture from metal fatigue; suit a human operator's physique 
and ability etc. The alien archaeologist would then come to the correct conclusion that we were an intelligent and creative species because our design details had a multiplicity of purpose. Some of the factors in the present paper suggest a "multiplicity of purpose". As an example, we might consider the value of the electronic charge which ensures the stability of matter at temperatures suitable for carbon-based life and also the rate of thermonuclear fusion in stars. In another example we might consider the mystery of gravity which allows animals to walk about safely on rocky planets and also allows stars to generate heat in a controlled manner.

It seems premature to clarify the issue of fine tuning. In the last paragraph of the book [11], Dawkins [21] wrote:

"Do you think some agent must have caused everything to start? Now we can read Lawrence Krauss for what looks to me like the knockout blow."........"

It seems appropriate to make a more moderating comment that we do not as yet have all the facts of the case before us and the Jury, i.e. the scientific community, has still to make a decision.

AII(c): Do other particles such as the electron have an inner structure like the proton?

Although electrons have a mass which is nearly 2,000 times smaller than protons there has long been speculation that electrons may also have an internal structure as yet unknown to us. Electrons are a major factor in Pauli's Exclusion Principle (PEP) which might be described as a very elaborate QSM. PEP is far more complicated than the new QSM described in section 4. PEP controls the electron's orbital limits in all atoms. It is reasonable to surmise some form of elaborate controlling communication between orbiting electrons and how they keep to their shells and the complications of having atomic nuclei of many different types and sizes.

An extensive recent European study [15] has confirmed experimentally that there is only a tiny probability (about 1 in $10^{29}$ ) that PEP will ever be violated naturally. This probability was assessed in an earlier study at 1 in $10^{27}$ but the new authors [15] predict that the probability of violation will eventually be certifiable as less than 1 in $10^{31}$. It seems that the matter control mechanisms described in this paper as QSMs can be very reliable indeed.

Unfortunately, electrons cannot be accelerated in a cyclotron as efficiently as the larger protons because they emit more braking radiation as they travel round bends. This energy-sapping braking radiation is usually called by its German name "brehmstrahlung" but other names are often used. Hesketh [3] calls it synchrotron radiation. The remedy to the energy loss problem is to use a linear accelerator but these are large and expensive. CERN are getting around the problem with a new type of plasma wave linear accelerator which is only $10 \mathrm{~m}$ long. This is well described with illustrations in [22] which must now be considered as an outdated report in this ongoing project. On request CERN will provide ongoing progress reports. Even if CERN's results from colliding electrons only confirm that electrons are true fundamental particles this would still be a most valuable contribution to our understanding.

\section{APPENDIX III: SEQUEL TO THE HISTORY OF GRANDMA'S CHAIR}

This addendum to the previous explanation [13] supports the conclusion that our Universe is a composite one containing both symmetrical and asymmetrical features.

Grandma did not quite reach her $90^{\text {th }}$ birthday, but her husband anticipating the event purchased a steel cylinder of compressed helium in order to inflate some balloons for her birthday party. Due to the sudden and unexpected loss of his wife the cylinder of helium was never opened and it just got stored in the cellar and forgotten. Grandpa went to live with his daughter and her family and the old rambling grandparent's house was let to many tenants as a rental property.

After several decades of decay, the old grandparents' house was purchased by a developer in order to build a block of flats. The workmen employed to demolish the old house discovered the old cylinder of helium now showing signs of severe corrosion. Clearly the cylinder was part of our asymmetric universe where time produces decay. Yet there was little evidence of the helium leaking from the cylinder. The atoms of helium had bounced off the inside of the cylinder for decades in the constant temperature of the cellar. Clearly the gas inside had behaved reversibly and elastically for decades with no entropy increase. It is reasonable to claim that the inside of the cylinder belonged to a symmetrical part of our Universe, yet the outside of the cylinder clearly belonged to an asymmetrical Universe.

Over the same period of time it is interesting to follow the history of Grandma's chair. Initially Grandma's daughter treated the chair as a valued family heirloom. It was preserved with furniture polish regularly and remained in near perfect condition. The daughter's children were never allowed to sit on it and the chair could be considered as a symmetrical part of our Universe just like the forgotten bottle of helium gas in the cellar of the old house. Over the years the children grew up and produced their own children and the daughter and her husband finally both died. The old chair soon became a forgotten part of family history. Finally, after years of neglect the chair was infected by woodworm. It had become part of our asymmetric Universe....... 